\title{
Connectivity Times for Mobile D2D Networks
}

\author{
Peter Smith \\ School of Mathematics and Statistics \\ Victoria University of Wellington \\ Wellington, New Zealand \\ peter.smith@vuw.ac.nz
}

\author{
Justin Coon \\ Department of Engineering Science \\ University of Oxford \\ Oxford, England \\ justin.coon@eng.ox.ac.uk
}

\begin{abstract}
Connectivity questions for mobile D2D networks are often approached by considering steady state performance metrics. This is due to the difficulty in handling a finite time horizon with random mobility. Hence, in this paper we create a framework to evaluate connectivity time in closed form over a finite time horizon for mobile devices. The basic metric is the mean proportion of time devices are connected over a finite period. The methodology is shown to deliver closed form results for a variety of deterministic and random mobility models in both fading and non-fading scenarios. This allows a comparison of mobility types and an understanding of the underlying parameters. In addition, an approach is developed which allows different mobility types to be compared on the basis of a single 'equivalent' speed and also the overall framework allows the energy requirements of certain mobility control mechanisms to be evaluated.
\end{abstract}

\section{INTRODUCTION}

Connectivity issues for random networks are well understood in the static case [1], but are more complex in mobile networks [2]. The effects of mobility are receiving increasing attention in the context of device-to-device (D2D) networks [3], [4], drone swarms [5] and IoT applications [6]. Hence, in this paper we are motivated to investigate a fundamental issue relating to mobile connectivity, namely, how long will two mobile devices be connected over a finite time horizon?

In particular, we fix a finite time period, $[0, T]$, and define a novel connectivity time as the expected proportion of time that two devices are connected in $[0, T]$. Connectivity is defined via a simple threshold signal-to-noise-ratio (SNR) value, links above the threshold are connected and those below are in outage. Clearly, the connectivity time depends on the device separation distance, which is governed by the mobility, environmental factors controlling the type of channel (fading and non-fading) and hardware issues such as transmit power. Hence, we parameterize the problem to take all these factors into account and consider both non-fading and Rayleigh fading scenarios.

Note that the finite time horizon is taken into account in a rather novel way, where $[0, T]$ is fixed and we derive the mean proportion of this interval where connectivity occurs. Hence, we are mixing average results with a finite interval. This approach is deliberate as it leads to tractable results. In contrast, it would be more direct to consider the distribution of the time connected in $[0, T]$. However, even for simple, stationary processes, such results are unavailable and complex approximations are the best solutions available to date [7].
A critical aspect of the connectivity problem is the type of mobility. Many mobility models have appeared [8], [9], [10] in order to investigate ad hoc networks, cellular communications, intelligent transportation systems, etc. Here, we consider five basic models which bracket a wide variety of behaviour. We consider two deterministic models: the static scenario and the case of linear motion at fixed speed and fixed direction. Three random models are also investigated. The first is a variation on linear mobility where the directions of the devices are random. The second is a two-dimensional Brownian motion process (2D BM) [11]. The final mobility type is for tethered devices where an Ornstein-Uhlenbeck (OU) process [11] models devices which drift in the environment, but have control mechanisms which attempt to return to the desired location. Although this paper focuses on the connectivity of two devices over a single-input single-output (SISO) link, extensions to multiple antennas and multiple users are possible. The main contributions of the paper are as follows:

- We create a framework to evaluate connectivity time in closed form over a finite time horizon for mobile devices.

- We compare the effects of five different types of mobility on connectivity time.

- We compute connectivity time for both fading and nonfading scenarios.

- We compare the effects of channel parameters, such as the path loss exponent, on connectivity time.

- We derive a framework for comparing different types of mobility by constructing an equivalent speed for each mobility type.

- We derive a simple, quantitative relationship between the steady-state connectivity time and key system parameters for the case where communicating devices experience positional perturbations corrected via feedback control.

\section{SySTEM MODEL}

Consider two mobile wireless devices moving in a twodimensional plane as shown in Fig.1. At time $t$, their separation is $d(t)$ with initial separation $d(0)=\Delta$. Assuming a SISO link between the two devices, the SNR of the link can be modelled in the classical way as:

$$
\gamma(t)=A L(t) d(t)^{-\eta}|h(t)|^{2},
$$

where $L(t)$ is shadowing, $\eta$ is the pathloss exponent, $h(t)$ is the fast fading normalized channel gain with $E\left[|h(t)|^{2}\right]=1$ 
and $A$ is a constant depending on transmit power, antenna properties, etc. Define connectivity as $\gamma(t)>\gamma_{0}$ for some connectivity threshold, $\gamma_{0}$, and the associated indicator function, $1(x)$, is given by $1(x)=1$ for $x>\gamma_{0}$ and $1(x)=0$ for $x \leq \gamma_{0}$. With these definitions, the proportion of time the two devices are connected in any finite time interval, $[0, T]$, is given by

$$
C_{T}=\frac{1}{T} \int_{0}^{T} 1(\gamma(t)) d t .
$$

The mean proportion of time the two devices are connected is therefore

$$
\begin{aligned}
\mu_{T} & =E\left[C_{T}\right]=\frac{1}{T} \int_{0}^{T} P\left(\gamma(t)>\gamma_{0}\right) d t \\
& =\frac{1}{T} \int_{0}^{T} P\left(A L(t) d(t)^{-\eta}|h(t)|^{2}>\gamma_{0}\right) d t .
\end{aligned}
$$

We refer to $\mu_{T}$ in (3) as the connectivity time. In this paper, we assume shadowing is fixed, $L(t)=L$ and there is either no fast fading or the fast fading is Rayleigh. Extensions to varying shadowing and other fading channels are left to further work. With these assumptions:

$$
\begin{aligned}
\mu_{T} & =\frac{1}{T} \int_{0}^{T} P\left(|h(t)|^{2}>\alpha d(t)^{\eta}\right) d t \\
& =\frac{1}{T} \int_{0}^{T} E\left[e^{-\alpha d(t)^{\eta}}\right] d t,
\end{aligned}
$$

where $\alpha=\gamma_{0} /(A L)$ using the fact that $|h(t)|^{2}$ is an exponential random variable and conditioning on the possibly random distance, $d(t)$. Clearly, connectivity time depends critically on the mobility model through the dependence on $d(t)$ in (4).

\section{A. Mobility Models}

In this work we consider five mobility models: labelled M1M5 for convenience. M1 is the default static scenario with no mobility. M2 assumes linear motion with constant speed where device $i$ has velocity $v_{i}$, with speed $\left|v_{i}\right|$ and a fixed angle, $\theta_{i}$, to the $x$-axis. M3 extends M2 to the case of random angles of direction. M4 considers that both devices follow a 2D BM path with no drift. Hence, M2, M3 and M4 are in some sense opposites as M4 has no trend whereas M2 and M3 are pure trend. M5 models the case where the devices are imperfectly tethered to a desired location. Hence, they drift randomly with a control mechanism that tends to push the device back to the target location. This is modelled by an OU process. In all cases, without loss of generality, we assume the initial position of device 1 is $(0,0)$ and of device 2 is $(0, \Delta)$.

\section{B. Separation distances}

Some straightforward calculations lead to the following separation distances for the five mobility models.

M1: $d(t)=\Delta \forall t$.

M2: $d(t)=\sqrt{\left(v_{x} t\right)^{2}+\left(\Delta+v_{y} t\right)^{2}}$, where $v_{x}=\left|v_{2}\right| \cos \left(\theta_{2}\right)-$ $\left|v_{1}\right| \cos \left(\theta_{1}\right)$ and $v_{y}=\left|v_{2}\right| \sin \left(\theta_{2}\right)-\left|v_{1}\right| \sin \left(\theta_{1}\right)$ are the $x$ and $y$ components of the velocity at which the devices move apart. M3: Separation distance is the same as for M2 except that $v_{x}$

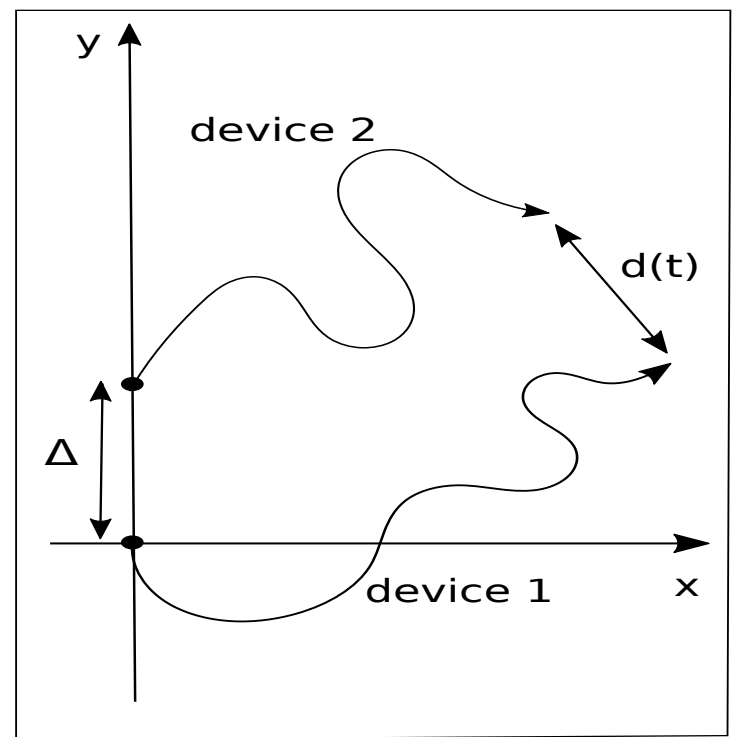

Figure 1. Schematic of device mobility

and $v_{y}$ are now random due to the random angles, $\theta_{1}$ and $\theta_{2}$. M4: Assuming drift free 2D BM with the same parameter for devices 1 and 2, we can write their positions at time $t$ as $\left(X_{1}(t), Y_{1}(t)\right)$ and $\left(X_{2}(t), \Delta+Y_{2}(t)\right)$ respectively where $X_{i}(t) \sim \mathcal{N}\left(0, \sigma^{2} t\right)$ and $Y_{i}(t) \sim \mathcal{N}\left(0, \sigma^{2} t\right)$ are iid Gaussian variables for $i=1,2$. Hence,

$$
\begin{aligned}
d(t) & =\sqrt{\left(X_{1}(t)-X_{2}(t)\right)^{2}+\left(\Delta+Y_{2}(t)-Y_{1}(t)\right)^{2}} \\
& =\sqrt{X^{2}(t)+(\Delta+Y(t))^{2}},
\end{aligned}
$$

where $X(t), Y(t)$ are independent $\mathcal{N}\left(0,2 \sigma^{2} t\right)$ variables and $\sigma^{2}$ controls the speed of the BM process.

M5: Assume identical zero mean OU processes [11] for the $\mathrm{X}$ - and y-coordinates for devices 1 and 2 . We can write their positions at time $t$ as $\left(X_{1}(t), Y_{1}(t)\right)$ and $\left(X_{2}(t), \Delta+Y_{2}(t)\right)$ respectively where $X_{i}(t) \sim \mathcal{N}\left(0, \frac{\sigma^{2}}{2 \theta}(1-\exp (-2 \theta t))\right)$ and $Y_{i}(t) \sim \mathcal{N}\left(0, \frac{\sigma^{2}}{2 \theta}(1-\exp (-2 \theta t))\right)$ are iid Gaussian variables for $i=1,2$. Hence, as in (5)

$$
d(t)=\sqrt{X^{2}(t)+(\Delta+Y(t))^{2}}
$$

where $X(t), Y(t)$ are independent $\mathcal{N}\left(0, \frac{\sigma^{2}}{\theta}(1-\exp (-2 \theta t))\right)$ variables. In this OU model, $\sigma^{2}$ controls the fluctuation in the position of the devices and $\theta$ is the rate of reversion of the device to the desired location. Note that when $\theta t$ is large, the OU process approaches a limiting distribution and $\operatorname{Var}[X(t)]=\operatorname{Var}[Y(t)] \rightarrow \sigma^{2} / \theta$. In contrast, the variance of the $\mathrm{BM}$ process continues to grow with $t$.

\section{Connectivity Times}

For the five mobility models, M1-M5, we now compute the connectivity time in (4) using the separation distances in Sec. II-B. 


\section{A. Mobility M1 (static)}

Since the separation distance is constant, $d(t)=\Delta$, (4) becomes

$$
\mu_{T}=e^{-\alpha \Delta^{\eta}}
$$

i.e., the connectivity time is simply the probability of connectivity.

\section{B. Mobility M2 (fixed linear mobility)}

Using the separation distance in Sec. II-B, the connectivity time in (4) becomes

$$
\mu_{T}=\frac{1}{T} \int_{0}^{T} \exp \left(-\alpha\left[\left(v_{x} t\right)^{2}+\left(\Delta+v_{y} t\right)^{2}\right]^{\eta / 2}\right) d t .
$$

The integral in (8) can be solved for $\eta=2$ since this makes the integrand a scaled version of a Gaussian density. For this scenario, using [12, Eq. 3.322(1)-(2)], we obtain

$$
\begin{aligned}
\mu_{T} & =\sqrt{\frac{\pi}{4 \alpha T^{2} v_{T}^{2}}} \exp \left(-\alpha \Delta^{2}+\frac{\alpha \Delta^{2} v_{y}^{2}}{v_{T}^{2}}\right) \times \\
& {\left[\operatorname{erf}\left(\frac{\sqrt{\alpha} \Delta v_{y}}{v_{T}}+T v_{T} \sqrt{\alpha}\right)-\operatorname{erf}\left(\frac{\sqrt{\alpha} \Delta v_{y}}{v_{T}}\right)\right], }
\end{aligned}
$$

where $v_{T}=\sqrt{v_{x}^{2}+v_{y}^{2}}$ and $\operatorname{erf}(\cdot)$ is the error function. The case of $\Delta=0$ can also be given in closed form since here,

$$
\begin{aligned}
\mu_{T} & =\frac{1}{T} \int_{0}^{T} \exp \left(-\alpha v_{T}^{\eta} t^{\eta}\right) d t \\
& =\frac{1}{v_{T} \eta T \alpha^{1 / \eta}} \gamma\left(\frac{1}{\eta}, \alpha v_{T}^{\eta} T^{\eta}\right),
\end{aligned}
$$

using [12] where $\gamma(\cdot, \cdot)$ is the incomplete gamma function [12, Eq. 7.550(1)]. For other parameter values, $\mu_{T}$ can be computed from (8) by numerical integration. Note that $\mu_{T}$ in (10) is $O\left(\frac{1}{T}\right)$ as $\gamma\left(1 / \eta, \alpha v_{T}^{\eta} T^{\eta}\right) \rightarrow \Gamma(1 / \eta)$ as $T \rightarrow \infty$.

\section{Mobility M3 (Variable linear mobility)}

From (4) and (8) we obtain

$$
\mu_{T}=E\left[\frac{1}{T} \int_{0}^{T} \exp \left(-\alpha\left[\left(v_{x} t\right)^{2}+\left(\Delta+v_{y} t\right)^{2}\right]^{\eta / 2}\right) d t\right] .
$$

Note that (11) involves three integrals, one over time and two to average over $\theta_{1}$ and $\theta_{2}$. For some scenarios it may be useful to interchange expectation and integration in (11). However, analytical progress is most likely for the $\Delta=0$ case where we can use (10) to give:

$$
\mu_{T}=\frac{1}{\eta T \alpha^{1 / \eta}} E\left[v_{T}^{-1} \gamma\left(1 / \eta, \alpha v_{T}^{\eta} T^{\eta}\right)\right] .
$$

Since $v_{T}=\sqrt{\left|v_{1}\right|^{2}+\left|v_{2}\right|^{2}-2\left|v_{1}\right|\left|v_{2}\right| \cos \left(\theta_{1}-\theta_{2}\right)}$, the expectation in (12) is a single integral over the variable $\theta_{1}-\theta_{2}$. For uniformly likely angles, $\theta_{i} \sim U[0,2 \pi], i=1,2$, the variable $X=\theta_{1}-\theta_{2}$ has the well-known triangular distribution:

$$
f_{X}(x)= \begin{cases}\frac{x+2 \pi}{4 \pi^{2}} & -2 \pi \leq x \leq 0 \\ \frac{-x+2 \pi}{4 \pi^{2}} & 0<x \leq 2 \pi\end{cases}
$$

and (12) becomes, after a change of variable,

$$
\mu_{T}=\frac{2 \alpha^{-1 / \eta}}{\eta T} \int_{0}^{1} \frac{x+1}{v_{T}(2 \pi x)} \gamma\left(\frac{1}{\eta}, \alpha v_{T}(2 \pi x)^{\eta} T^{\eta}\right) d x,
$$

using the notation $v_{T}(x)=\sqrt{\left|v_{1}\right|^{2}+\left|v_{2}\right|^{2}-2\left|v_{1}\right|\left|v_{2}\right| \cos (x)}$. All functions in (14) are smooth and well-behaved and the integral is finite range leading to a simple, single numeric integration solution. As in (10), connectivity time is also $O\left(\frac{1}{T}\right)$ for M3. For the majority of other parameter values, $\mu_{T}$ can be computed from (11) by numerical integration.

\section{Mobility M4/M5 (Brownian Motion/OU Processes)}

For both these models, the Gaussian processes $\mathrm{X}(\mathrm{t})$ and $\mathrm{Y}(\mathrm{t})$ defined in (5) and (6) can be redefined as $X(t)=c(t) U(t)$ and $Y(t)=c(t) V(t)$ at any point in time, where $U(t), V(t)$ are iid $\mathcal{N}(0,1)$ variables. For M4, $c^{2}(t)=2 \sigma^{2} t$, and for M5, $c^{2}(t)=\sigma^{2}(1-\exp (-2 \theta t)) / \theta$. Using the separation distances in (5) and (6), the connectivity time, $\mu_{T}$, in (4) becomes

$$
\frac{1}{T} \int_{0}^{T} E\left[\exp \left(-\alpha\left[c^{2}(t) U^{2}(t)+(\Delta+c(t) V(t))^{2}\right]^{\frac{\eta}{2}}\right)\right] d t .
$$

For any instant in time, $W(t)=U^{2}(t)+(\Delta / c(t)+V(t))^{2}$ has a non-central Chi-squared distribution with two degrees of freedom and non-centrality parameter, $\delta(t)=\Delta^{2} / c^{2}(t)$, ie., $W(t) \sim \chi_{2}^{2}(\delta(t))$. Hence, using the PDF of $W(t)$ given in [13], (15) becomes

$$
\int_{0}^{T} \int_{0}^{\infty} \frac{\exp \left(-\alpha c^{\eta}(t) w^{\eta / 2}-\frac{(w+\delta(t))}{2}\right) I_{0}(\sqrt{w \delta(t)})}{2 T} d w d t
$$

For $\eta=2$, integration over $w$ in (16) is possible using [12, Eq. 6.614(3)], giving

$$
\int_{0}^{T} \frac{1}{T} \exp \left(\frac{-\alpha c^{2}(t) \delta(t)}{2 \alpha c^{2}(t)+1}\right) \frac{1}{2 \alpha c^{2}(t)+1} d t .
$$

Also, for $\Delta=0$, (16) simplifies to

$$
\frac{1}{2 T} \int_{0}^{T} \int_{0}^{\infty} \exp \left(-\alpha c^{\eta}(t) w^{\eta / 2}-w / 2\right) d w d t
$$

For $\eta=2$, (18) collapses to

$$
\frac{1}{T} \int_{0}^{T} \frac{1}{2 \alpha c^{2}(t)+1} d t
$$

which agrees with (17) when $\delta(t)=0$. For $\eta=4$, integrating (18) over $w$ using [12, Eq. 3.322(2)] gives

$$
\frac{\sqrt{\pi}}{4 T \sqrt{\alpha}} \int_{0}^{T} \frac{1}{c^{2}(t)} \exp \left(\frac{1}{16 \alpha c^{4}(t)}\right)\left(1-\Phi\left(\frac{1}{4 \sqrt{\alpha} c^{2}(t)}\right)\right)_{(20)} d t .
$$

Results (16), (17), (18) and (20) are valid for both M4 and M5 and no further simplifications seem to be available for M5. However, for 2D BM (M4), (18) reduces to

$$
\mu_{T}=\frac{1}{\eta T} \int_{0}^{\infty} \frac{\exp (-w / 2)}{2 \sigma^{2} w \alpha^{2 / \eta}} \gamma\left(\frac{2}{\eta}, \alpha\left(2 \sigma^{2} w T\right)^{\eta / 2}\right) d w,
$$

and (19) reduces to

$$
\mu_{T}=\frac{\log \left(1+4 \alpha \sigma^{2} T\right)}{4 \alpha \sigma^{2} T},
$$


using the definition of $\gamma(\cdot, \cdot)$ and simple integration rules. In contrast to M2 and M3, where $\mu_{T}$ is $O\left(\frac{1}{T}\right)$, from (22) we see that $\mu_{T}$ is $O\left(\frac{\log (T)}{T}\right)$. Also, for M5, (19) simplifies to

$$
\begin{aligned}
\mu_{T} & =\log ((1+\kappa) \exp (2 \theta T)-\kappa) /(2 \theta T(1+\kappa)) \\
& =(1+\kappa)^{-1}+O(1 / T),
\end{aligned}
$$

where $\kappa=2 \alpha \sigma^{2} / \theta$. Hence, the single parameter, $\kappa$, controls long term behaviour and has applications to communication subsystem design in, e.g., robotic swarms or D2D UAV networks subject to positional perturbations.

\section{E. The No-Fading Case}

In the absence of fading, i.e $|h(t)|^{2}=1$, the only randomness is due to separation distance. Hence, M1 and M2 mobility scenarios lead to deterministic connectivity times. As a result, we focus on M3-M5 and further assume that $L(t)=1, \eta=2$, which models free space LOS signals with no fading. In this scenario, from (3), we have:

$$
\mu_{T}=\frac{1}{T} \int_{0}^{T} P\left(d^{2}(t)<\frac{A}{\gamma_{0}}\right) d t .
$$

For mobility scenario $\mathrm{M} 3, \mu_{T}$ is found from (24) using the separation distance in Sec. II-B and treating the angles $\theta_{1}$ and $\theta_{2}$ as random. This simplifies if $\Delta=0$ since here (24) is a function of the single variable, $v_{T}$, rather than both $\theta_{1}$ and $\theta_{2}$. Hence, the PDF in (13) can be used. For M4 and M5, the more interesting cases, we have

$$
\mu_{T}=\frac{1}{T} \int_{0}^{T} P\left(c^{2}(t) U^{2}(t)+(\Delta+c(t) V(t))^{2}<\frac{A}{\gamma_{0}}\right) d t .
$$

Rewriting (25) using the non-central $\chi^{2}$ variable, $W(t)$, defined in Sec. III-D gives

$$
\begin{aligned}
\mu_{T} & =\frac{1}{T} \int_{0}^{T} P\left(c^{2}(t) W(t)<\frac{A}{\gamma_{0}}\right) d t \\
& =\frac{1}{T} \int_{0}^{T} F_{2, \delta(t)}\left(\frac{A}{\gamma_{0} c^{2}(t)}\right) d t,
\end{aligned}
$$

where $F_{2, \delta(t)}(\cdot)$ is the CDF of a non-central $\chi^{2}$ variable with two degrees of freedom and non-centrality parameter, $\delta(t)$ [13]. For $\Delta=0, W(t)$ collapses to $W(t)=U^{2}(t)+V^{2}(t)$, an exponential with mean 2. Here, (26) simplifies giving:

$$
\mu_{T}=\frac{1}{T} \int_{0}^{T} 1-\exp \left(\frac{-A}{2 c^{2}(t) \gamma_{0}}\right) d t .
$$

For M4, using [12, Eq. 3.351(4)], (28) can be computed as:

$$
\mu_{T}=1-\exp \left(\frac{-A}{4 \sigma^{2} T \gamma_{0}}\right)+\frac{A}{4 \sigma^{2} T \gamma_{0}} E_{1}\left(\frac{A}{4 \sigma^{2} T \gamma_{0}}\right) \text {. }
$$

Similarly, for M5, integrating (28) gives

$$
\begin{aligned}
\mu_{T} & =1+\frac{1}{2 \theta T} E_{1}\left(\frac{\zeta}{1-e^{-2 \theta T}}\right)-\frac{\exp (-\zeta)}{2 \theta T} E_{1}\left(\frac{\zeta e^{-2 \theta T}}{1-e^{-2 \theta T}}\right) \\
& =1-\exp (-\zeta)+O(1 / T),
\end{aligned}
$$

where $\zeta=A \theta /\left(2 \gamma_{0} \sigma^{2}\right)$ is a single parameter which parameterizes the long term behaviour of $\mu_{T}$.

\section{NUMERICALRESULTS}

\section{A. Parameter values}

The parameters controlling the channel, time period, connectivity and initial position are $A, L, \gamma_{0}, T, \eta$ and $\Delta$. From (4) we see that the effects of are $A, L, \gamma_{0}$ are controlled by $\alpha$. Also, $T=1$ without loss of generality as it is the speed of the devices relative to $T$ that is important rather than the absolute value of $T$. Hence, the parameter values reduce to $\alpha$, $\eta$ and $\Delta$. Now $\eta$ and $\Delta$ are set according to various scenarios in each figure, so only the value of $\alpha$ remains to be discussed, which follows after a description of the mobility parameters.

The parameters controlling mobility are the speeds $\left(\left|v_{1}\right|\right.$ and $\left.\left|v_{2}\right|\right)$ and directions ( $\theta_{1}$ and $\left.\theta_{2}\right)$ for M2 and M3 and the parameters $\sigma^{2}$ for the 2D BM (M4) and $\theta, \sigma^{2}$ for the OU process (M5). For the linear mobility cases we select a constant speed for each device so that $s=\left|v_{1}\right|=\left|v_{2}\right|$ and $s$ is given in each figure. The angles are either fixed according to a given scenario (M2) or iid, $\theta_{i} \sim U[0,2 \pi], i=1,2$, for M3. For M2 we give results for two particular sets of directions. These are labeled: perp (where the two devices move perpendicularly to one another, $\theta_{1}=0$ and $\theta_{2}=\pi / 2$ ) and opp (where the two devices move directly away from one another, $\theta_{1}=-\pi / 2$ and $\theta_{2}=\pi / 2$ ). The parameter $\sigma^{2}$ controls the size of the random fluctuations in the device position over time for both BM and OU processes. For the OU process, the parameter $\theta$ controls the rate at which the device reverts back to the desired location (the initial position). Physically, the instantaneous velocity of the device in both $\mathrm{x}$ and $\mathrm{y}$-directions towards the intial point is given by $\theta \times$ displacement.

Where the different mobility models are compared it is necessary to match the speeds of the devices in some way. For M2 and M3 the speed of a device is fixed at $s$. Hence, in time $T$, the devices move a distance $s T$. To give a similar movement for the random BM and OU processes in the same time frame we set the mean time for the device to reach distance $s T$ from the initial point to be $T$. If $T_{s}$ is the time taken for a random process to first reach a distance $s T$ from the initial position, then we require $E\left[T_{s}\right]=T$. A methodology for computing $E\left[T_{s}\right]$ is given in [11] for both $\mathrm{OU}$ and 2D BM processes. Following this method gives

$$
E\left[T_{s}\right]=\frac{(s T)^{2}}{2 \sigma^{2}}=T,
$$

for 2D BM (M4) and for M5, $E\left[T_{s}\right]=T$ gives

$$
\frac{1}{2 \theta}\left(-\operatorname{Re}\left(\mathrm{E}_{1}\left(-\frac{\theta(s T)^{2}}{\sigma^{2}}\right)\right)-\log \left(\frac{\theta(s T)^{2}}{\sigma^{2}}\right)-\gamma\right)=T,
$$

where $\gamma$ is Euler's constant. Hence the value of $\sigma^{2}$ is $s^{2} T / 2$ for BM and for the OU process (32) is solved numerically for $\theta, \sigma^{2}$ using $\theta=1$ unless otherwise stated.

Lastly, we consider the value of $\alpha$ which is set relative to a reference speed of $s=1$ for linear mobility. The value of $\alpha$ is chosen so that a target probability of connectivity $\left(P_{\text {target }}\right)$ is achieved at time $T=1$ in a Rayleigh fading channel. Hence, using (4) we have $\exp (-\alpha)=P_{\text {target }}$ so that $\alpha=-\log \left(P_{\text {target }}\right)$. 


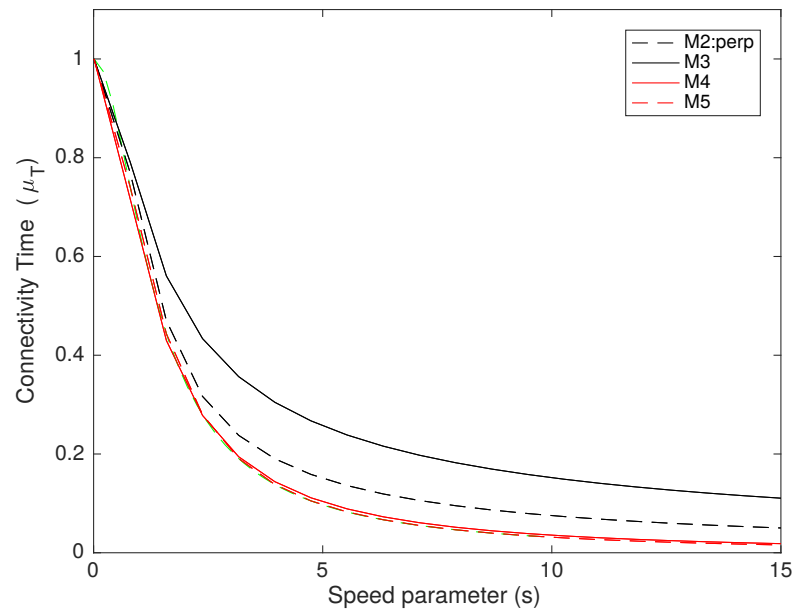

Figure 2. Connectivity time vs speed parameter for M2, M3, M4 and M5 with $P_{\text {target }}=0.5 ; T=1 ; \Delta=0 ; \eta=2$ and Rayleigh fading

\section{B. Discussion}

Note that all results given in Figs. 2-6 have been verified by numerical simulation of (3) and (24) for the fading and non-fading cases, respectively. Figure 2 shows connectivity time vs the speed parameter for M2 (using (9)), M3 (using (14)), M4 (using (22)) and M5 (using (19) in the Rayleigh fading scenario. Note that the method used in (31) and (32) is very effective at giving comparable rates of change (or speeds) for the different mobility processes. Hence, the connectivity time behaviour is quite similar even across the very different mobility types. Since $\Delta=0$, the devices are always connected if stationary and hence, the curves drop off from $\mu_{T}=1$ at $s=0$. For larger speeds, M3 gives higher connectivity than M2: perp because with M3 there is a chance that the two devices diverge more slowly than perpendicular motion. M4 and M5 are very similar, although as expected, the tethered OU process has slightly higher connectivity. Figure 3 shows connectivity time vs the speed parameter for M2 (using (24)), M4 (using (29)) and M5 (using (28)) in the non-fading scenario. In the absence of fading, connectivity is almost certain for low speeds over $T=1$ and so the curves are much flatter at the origin than in the fading case shown in Fig. 2. Again, M4 and M5 are very similar and for M2, perpendicular motion gives higher connectivity than opposite. Figure 4 shows connectivity time vs the speed parameter for M2 (using (9)), M4 (using (17)) and M5 (using (17)) in the Rayleigh fading scenario. The effect of $\Delta$ is clear, with increasing initial separation leading to reduced conectivity. Again, perpendicular motion gives higher connectivity than opposite motion. Note that the initial decay is much slower for M4 and M5, because M2 always gives increasing separation over time and so the connectivity curves decay quickly. In contrast, for non-zero values of $\Delta$ the random mobilities in M4 and M5 have some probability of reducing separation and this makes the decay at small speeds more gradual and leads to M4 and M5 giving

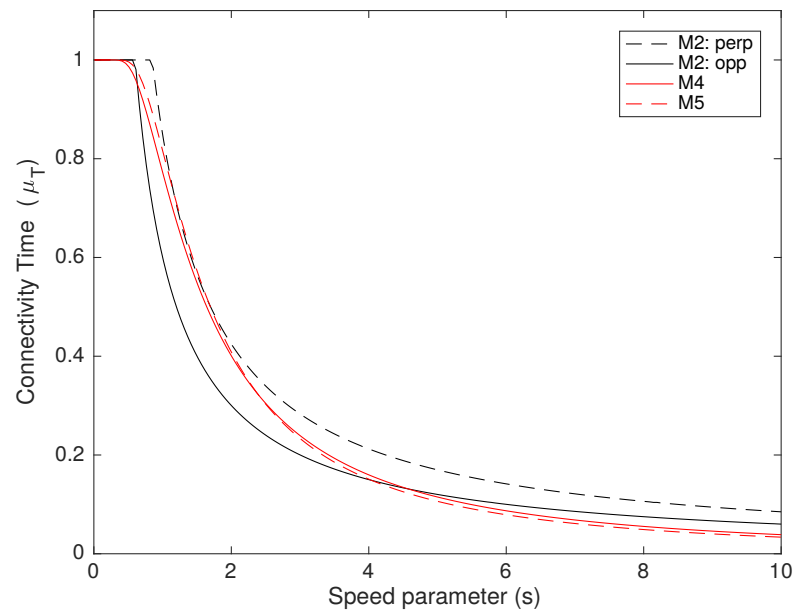

Figure 3. Connectivity time vs speed parameter for M2 (with two scenarios), M4 and M5 with $P_{\text {target }}=0.5 ; T=1 ; \Delta=0 ; \eta=2$ and no fading

higher connectivities than M2.

Figure 5 shows connectivity time vs elapsed time for M4 (using (17)) and M5 (using (17)) in the Rayleigh fading scenario. Here, the effect of the $\theta$ parameter is explored for the OU process. Since $\theta$ controls the rate at which the devices revert to their original location, the larger the value of $\theta$ the higher the connectivity and as $\theta \rightarrow 0$, the results for the OU process converge to those of the BM process. The stabilizing property of the OU process is shown in Fig. 5, where, as $\theta$ grows larger, a limiting distribution is approached and the connectivity times reach a constant level, which matches $1 /(1+\kappa)$ derived in (23). Of course, the price to be paid for greater control is that increasing $\theta$ increases the energy requirements of the device. Since, the velocity supplied by the control mechanism is $\theta \times$ displacement in both dimensions, the kinetic energy supplied for a device of mass $m$ at time $t$ is $\frac{1}{2} m\left[(\theta X(t))^{2}+(\theta Y(t))^{2}\right]$. Hence, the mean energy requirement, $E_{T}$, over the time period $[0, T]$ is

$$
\begin{aligned}
E_{T} & =E\left[\int_{0}^{T} \frac{1}{2} m \theta^{2}\left(X^{2}(t)+Y^{2}(t)\right) d t\right] \\
& =\frac{m \sigma^{2}}{4}[2 \theta T+\exp (-2 \theta T)-1],
\end{aligned}
$$

using $E\left[X^{2}(t)\right]=\frac{\sigma^{2}}{2 \theta}(1-\exp (-2 \theta t))$. For moderate and large values of $\theta T$ we observe that $E_{T}$ grows linearly with $\theta, T, m$, and $\sigma^{2}$. Computation of $\operatorname{Var}[T]$ are also possible, which will help characterise the likelihood of high energy requirements. Figure 6 shows connectivity time vs elapsed time for a subset of the cases in Fig. 5 and two different $\eta$ values, $\eta=2$ and $\eta=$ 4. As expected, as speed increases and the distances travelled grow larger, the effects of increased path loss are to reduce connectivity time. The impact on connectivity time is smaller for $P_{\text {target }}=0.5$ and more pronounced for $P_{\text {target }}=0.5$. Hence, as $\alpha=-\log \left(P_{\text {target }}\right)$ becomes smaller the effect of the path loss exponent is greater. This trend can be identified from (4) 


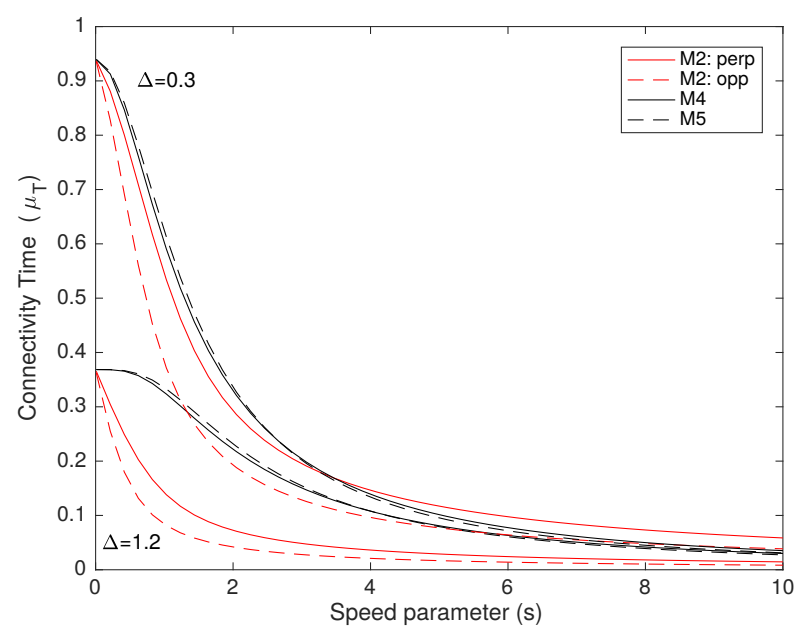

Figure 4. Connectivity time vs speed parameter for M2 (with two scenarios), M4 and M5 with $P_{\text {target }}=0.5 ; T=1 ; \eta=2$ and Rayleigh fading

by noting that larger $\alpha$ values reduce the connectivity time to small values where the effects of $\eta$ are necessarily small. However, for small $\alpha$, larger connectivity times are possible where the effects of $\eta$ can be seen more obviously.

\section{Conclusions}

In this paper, we define the concept of a finite time horizon connectivity metric. This leads to a tractable mathematical approach and is applied to five different types of mobility. The mobility scenarios cover a range of scenarios from deterministic linear motion to random walks and tethered devices with random wander. The connectivity time is derived and computed for both fading and non-fading scenarios allowing a comparison of the effects of channel parameters on connectivity time. Further, we derive a framework for comparing different types of mobility by constructing an equivalent speed for each type of motion. Finally, we note that the framework allows an investigation of the energy requirements of certain mobility control mechanisms.

\section{REFERENCES}

[1] M. Xiang, L. Sun, and L. Li, "Survey on the connectivity and coverage in wireless sensor networks," in 2011 7th International Conference on Wireless Communications, Networking and Mobile Computing, Sept 2011, pp. 1-4.

[2] A. Asadi, Q. Wang, and V. Mancuso, "A survey on device-to-device communication in cellular networks," IEEE Communications Surveys Tutorials, vol. 16, no. 4, pp. 1801-1819, Fourth quarter 2014.

[3] D. Wu et al., "The role of mobility for D2D communications in LTEadvanced networks: Energy vs. bandwidth efficiency," IEEE Wireless Communications, vol. 21, no. 2, pp. 66-71, April 2014.

[4] R. Wang, J. Zhang, S. H. Song, and K. B. Letaief, "Mobility increases the data offloading ratio in D2D caching networks," in 2017 IEEE International Conference on Communications, May 2017, pp. 1-6.

[5] V. Autefage, S. Chaumette, and D. Magoni, "A mission-oriented service discovery mechanism for highly dynamic autonomous swarms of unmanned systems," in 2015 IEEE International Conference on Autonomic Computing, July 2015, pp. 31-40.

[6] D. Wu et al., "UbiFlow: Mobility management in urban-scale software defined IoT," in 2015 IEEE Conference on Computer Communications (INFOCOM), April 2015, pp. 208-216.

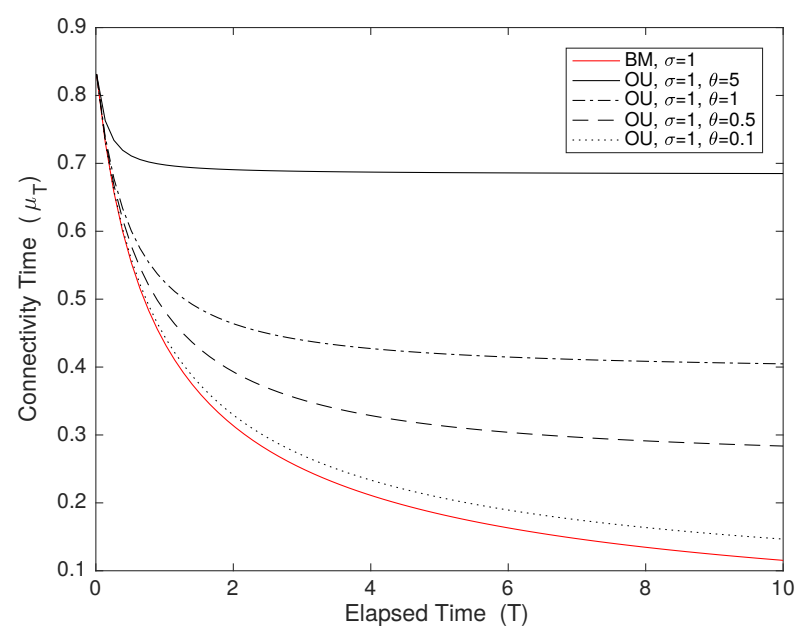

Figure 5. Connectivity time vs elapsed time ( $T$ ) for M4 and M5 with $P_{\text {target }}=$ $0.5 ; \eta=2, \Delta=0.5$ and Rayleigh fading

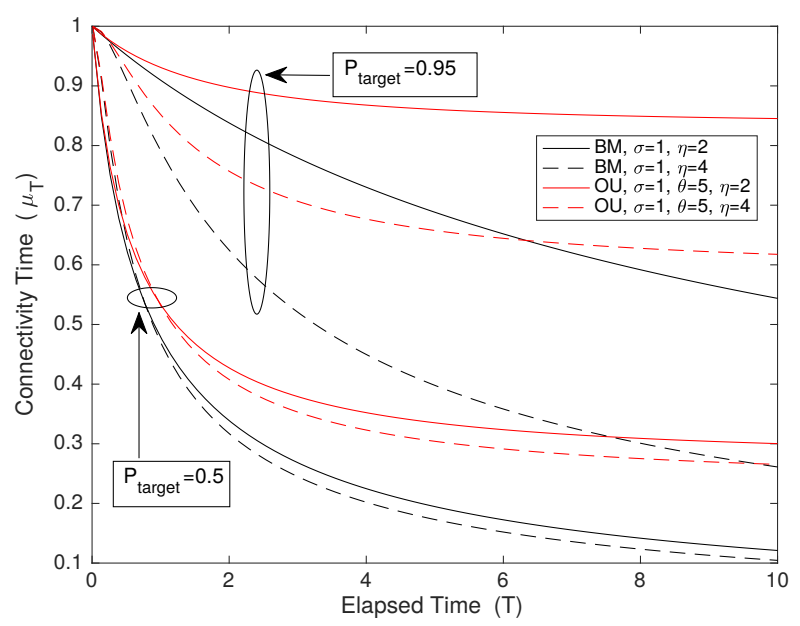

Figure 6. Connectivity time vs elapsed time ( $T$ ) for M4 and M5 with $P_{\text {target }} \in$ $\{0.5,0.95\} ; \Delta=0$ and Rayleigh fading

[7] F. A. Ramos, V. Y. Kontorovitch, and M. Lara, "On the fade duration distribution in Nakagami fading channels," in 2007 IEEE International Conference on Communications, June 2007, pp. 5052-5057.

[8] C. Bettstetter, G. Resta, and P. Santi, "The node distribution of the random waypoint mobility model for wireless ad hoc networks," IEEE Transactions on Mobile Computing, vol. 2, no. 3, pp. 257-269, July 2003.

[9] M. M. Zonoozi and P. Dassanayake, "User mobility modeling and characterization of mobility patterns," IEEE Journal on Selected Areas in Communications, vol. 15, no. 7, pp. 1239-1252, Sep 1997.

[10] J. Harri, F. Filali, and C. Bonnet, "Mobility models for vehicular ad hoc networks: A survey and taxonomy," IEEE Communications Surveys Tutorials, vol. 11, no. 4, pp. 19-41, Fourth 2009.

[11] C. Gardiner, Stochastic Methods: A Handbookfor the Natural and Social Sciences. Springer-Verlag, 2009.

[12] I. Gradshteyn, A. Jeffrey, and I. Ryzhik, Table of Integrals, Series, and Products. Academic Press, 1996.

[13] N. Johnson, S. Kotz, and N. Balakrishnan, Distributions in Statistics: Continuous Univariate Distributions-2. John Wiley and Sons, 1970. 